\title{
Robust Fault-Tolerant Control for Uncertain Networked Control Systems with State-Delay and Random Data Packet Dropout
}

\author{
Xiaomei Qi, ${ }^{1}$ Chengjin Zhang, ${ }^{1}$ and Jason $\mathbf{G u}^{2}$ \\ ${ }^{1}$ School of Control Science and Engineering, Shandong University, Jinan 250061, China \\ ${ }^{2}$ Department of Electrical and Computer Engineering, Dalhousie University, Halifax, NS, Canada B3J 2 X4
}

Correspondence should be addressed to Chengjin Zhang, cjzhang@sdu.edu.cn

Received 22 November 2011; Accepted 9 February 2012

Academic Editor: Yang Shi

Copyright ( 2012 Xiaomei Qi et al. This is an open access article distributed under the Creative Commons Attribution License, which permits unrestricted use, distribution, and reproduction in any medium, provided the original work is properly cited.

\begin{abstract}
A robust fault-tolerant controller design problem for networked control system (NCS) with random packet dropout in both sensor-to-controller link and controller-to-actuator link is investigated. A novel stochastic NCS model with state-delay, model uncertainty, disturbance, probabilistic sensor failure, and actuator failure is proposed. The random packet dropout, sensor failures, and actuator failures are characterized by a binary random variable. The sufficient condition for asymptotical mean-square stability of NCS is derived and the closed-loop NCS satisfies $H_{\infty}$ performance constraints caused by the random packet dropout and disturbance. The fault-tolerant controller is designed by solving a linear matrix inequality. A numerical example is presented to illustrate the effectiveness of the proposed method.
\end{abstract}

\section{Introduction}

Networked control systems (NCSs) are one type of distributed control systems, in which the information of control system components (sensors, controllers, actuators, etc.) is exchanged via communication networks (see Figure 1). Compared with the conventional point-to-point control systems, NCSs have many advantages, such as low cost of installation and maintenance, ease of diagnosis, and flexible architectures. However, the network in the control systems also bring many problems, such as network-induced delay, packet dropout, multiple channel transmission (as in [1-4]). Recent researches have a deep look into the controller and filter design for NCSs without faults (see [5-10]) and the references therein.

Actually, NCSs are more vulnerable to faults than conventional control systems, due to the complexity introduced by the network. It is very significant to guarantee security and reliability of NCSs, because modern technological systems rely on sophisticated control systems to meet increased performance and safety requirement. Therefore, research on fault-tolerant control (FTC) for NCSs has attracted more and more attention from both industry and academia.
However, research on FTC for NCSs is quite different from the ones for conventional control systems in many aspects (see [11-15]). A suitable architecture for FTC of NCSs must take into consideration the dynamical behaviour of network. In most research on FTC for NCSs, the fault model is described in a static way (as in [16-20]). Actually, faults often happen in a random way, so it is suitable to be studied in a dynamic way (as in [21-25]). In [21], the probabilistic sensor reductions are modeled by using a random variable that obeys a specific distribution in a known interval. In [22], the entire sensor failures or missing measurements have been described as a Bernoulli distributed variable. The reliable control design is considered for NCSs against probabilistic actuator fault with different failure rates in [23]. But, only actuator failures or sensor failures are considered in [16-23].

As we know, many engineering control systems such as conventional oil-chemical industrial processes, nuclear reactors, long transmission lines in pneumatic, hydraulic, and rolling mill systems, NCSs contain some time-delay effects, model uncertainties (as in $[26,27]$ ), and external disturbances. However, most of the aforementioned researches discuss FTC for NCSs without model uncertainty and disturbance(as in [16-20, 23, 24]). 


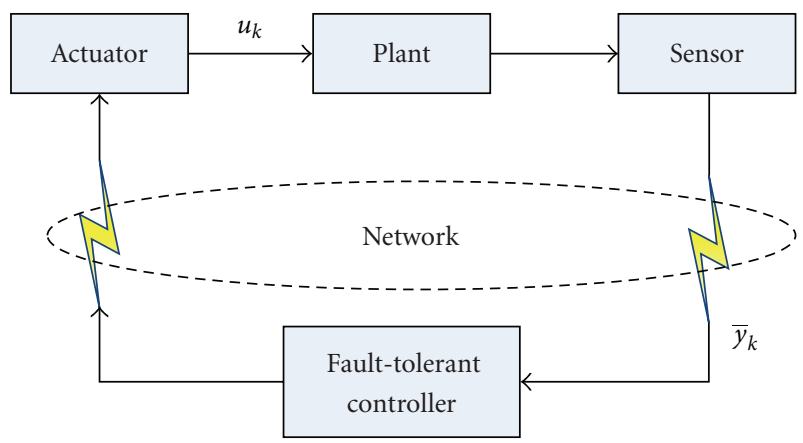

FIGURE 1: Schematic description of a distributed NCS.

Therefore, from the above description, considering random packet dropout, the robust FTC for state-delay uncertain NCSs with both probabilistic sensors failures and actuators failures is still a challenging problem.

In this paper, we study the robust FTC problem for NCSs with random packet dropout in both sensor-to-controller (S-C) link and controller-to-actuator (C-A) link. A new stochastic NCS model with fault is proposed, which includes the state-delay, model uncertainty, disturbance, random packet dropout, probabilistic sensors failures, and actuators failures. The random packet dropout, the sensor failure and the actuator failure are described as a binary random variable. The aim of this paper is to design a dynamic faulttolerant controller for the NCS including packet dropouts, both sensor failures and actuator failures. The closed-loop NCS can be asymptotical mean-square stability and satisfies the performance constraint.

The rest of the paper is organized as follows. The problem is formulated in Section 2, a new stochastic NCS with probabilistic sensors failures and actuators failures is modelled. Section 3 presents the integral analysis of asymptotical meansquare stability for stochastic NCS with sensor and actuator faults. Section 4 designs a dynamic fault-tolerant controller. Section 5 gives a numerical example to demonstrate the effectiveness of the proposed method. Concluding remarks are made in Section 6.

\section{Problem Formulation}

Consider the following uncertain linear state-delay system:

$$
\begin{gathered}
x_{k+1}=(A+\Delta A) x_{k}+A_{d} x_{k-d}+B u_{k}+D_{0} \omega_{k}, \\
y_{k}=C_{0} x_{k}, \\
z_{k}=C x_{k}+C_{d} x_{k-d}+D_{1} \omega_{k},
\end{gathered}
$$

where $x_{k} \in \mathbb{R}^{n}, y_{k} \in \mathbb{R}^{m}, u_{k} \in \mathbb{R}^{p}, z_{k} \in \mathbb{R}^{q}$ denote the state, the sensor measurement, the control input, and the controlled output, respectively. $\omega_{k} \in \mathbb{R}^{r}$ is the disturbance input belonging to $l_{2}[0, \infty) . A, A_{d}, B, C_{0}, C, C_{d}, D_{0}, D_{1}$, are known real constant matrices with appropriate dimensions. $d>0$ is a known delay. $\Delta A$ denotes the model uncertainty, which satisfies $\Delta A=H F_{k} N, F_{k}^{T} F_{k}<I, F_{k}$ represents an unknown real-valued time-varying matrix.
Figure 1 shows a typical feedback loop of NCS. Due to network congestion, traffic load balancing, or other unpredictable network behavior, the network-induced delay, data packet dropout, disorder may occur at the same time. In this paper, we focus on the data packet dropout phenomenon. Some assumptions in this paper are as follows.

(1) The sensor is clock-driven, the controller and the actuator are event-driven.

(2) Data packet dropouts occur in both S-C link and C-A link.

(3) Data are single-packet transmission with timestamp.

(4) Ignore the effects of quantization and asynchronous error in this paper.

Remark 1. A clock-driven sensor can send measurements to network periodically and is often used in real-time computing. The advantage of event-driven controller/actuator is that the controller/actuator will be updated as soon as the new data packet comes.

Remark 2. Taking the Internet as an illustration, the Transmission Control Protocol (TCP) is one of the core protocols of the Internet Protocol Suite. TCP is responsible for verifying the correct delivery of data from client to server. Data can be lost in the intermediate network. TCP adds support to detect errors or lost data and to trigger retransmission until the data is correctly and completely received. Thus, TCP is optimized for accurate delivery rather than timely delivery. Furthermore, for real-time feedback control, it is appropriate to discard the old data and transmit a new packet if it is available.

Therefore, we assume if the total network-induced delay $\tau_{k}$ is larger than a sampling period, the output terminal will actively discard this packet, which means the networkinduced delay problem can be considered as a packetdropout problem. And the receiver with a buffer can rearrange the packets by reading the information of timestamp; in this way, the data disorder problem will be solved. Hence, we only focus on the data packet dropout issue in this paper.

The binary random variable $\theta_{k}$ is an identically distributed (i.i.d.) process. $\theta_{k}=1$ means that there is no packet dropout, and the sensors and the actuators are reliable; $\theta_{k}=$ 0 means packet is lost, and the sensors and the actuators have failures. The probability distribution of $\theta_{k}$ is $P\left\{\theta_{k}=0\right\}=$ $p, P\left\{\theta_{k}=1\right\}=1-p$, where $p \in(0,1)$ indicates the sensor /actuator failure rate and the packet dropout rate.

Considering the channel from the sensor to the controller, the sensor measurement $y_{k}$ will be updated to $\bar{y}_{k}$ as follows:

$$
\bar{y}_{k}=\theta_{k} y_{k}+\left(1-\theta_{k}\right) \bar{y}_{k-1} .
$$

Considering the channel from the controller to the actuator, the control output $u_{k}$ will be updated as follows:

$$
u_{k}=\theta_{k} u_{k}+\left(1-\theta_{k}\right) u_{k-1} .
$$


Remark 3. From expressions (2) and (3), when $\theta_{k}=0$ at time $k$, the sensor measurements and control information at time $k$ are missing. The last available measurement $\bar{y}_{k-1}$ and controller output $u_{k-1}$ stored in a buffer are utilized to substitute the missing data, which means at least one packet can be transmitted successfully in a sampling period.

Since the random variable $\theta_{k}$ also represents sensor failures and actuator failures, the dynamic fault-tolerant controller is designed

$$
\begin{gathered}
\hat{x}_{k+1}=A_{f} \hat{x}_{k}+B_{f} \bar{y}_{k}, \\
u_{k}=C_{f} \hat{x}_{k} .
\end{gathered}
$$

Define $\eta_{k}=\left[x_{k}^{T}, \hat{x}_{k}^{T}\right]^{T}, d_{k}=\left[u_{k-1}^{T}, \bar{y}_{k-1}^{T}\right]^{T}$, the stochastic NCS with probabilistic sensor failures and actuator failures

$$
\begin{aligned}
\eta_{k+1}= & \tilde{A} \eta_{k}+\left(\theta_{k}-p\right) \tilde{A}_{2} \eta_{k}+\tilde{A}_{d} Z \eta_{k-d} \\
& +\left(\theta_{k}-p\right) \widetilde{B}_{1} d_{k}+\widetilde{B}_{2} d_{k}+\widetilde{D}_{1} \omega_{k}, \\
z_{k}= & \widetilde{C} \eta_{k}+C_{d} Z \eta_{k-d}+D_{1} \omega_{k},
\end{aligned}
$$

where

$$
\begin{gathered}
\widetilde{A}=\widetilde{A}_{1}+\tilde{H} F_{k} \tilde{N}=\left[\begin{array}{cc}
A & p B C_{f} \\
p B_{f} C_{0} & A_{f}
\end{array}\right]+\left[\begin{array}{c}
H \\
0
\end{array}\right] F_{k}\left[\begin{array}{ll}
N & 0
\end{array}\right], \\
\widetilde{A}_{2}=\left[\begin{array}{cc}
0 & B C_{f} \\
B_{f} C_{0} & 0
\end{array}\right], \quad \widetilde{A}_{d}=\left[\begin{array}{c}
A_{d} \\
0
\end{array}\right], \\
\widetilde{B}_{1}=\left[\begin{array}{cc}
-B & 0 \\
0 & -B_{f}
\end{array}\right], \quad \widetilde{B}_{2}=\left[\begin{array}{cc}
(1-p) B & 0 \\
0 & (1-p) B_{f}
\end{array}\right], \\
\widetilde{C}=\left[\begin{array}{ll}
C & 0
\end{array}\right], \quad \widetilde{D}_{1}=\left[\begin{array}{c}
D_{0} \\
0
\end{array}\right], \quad Z=\left[\begin{array}{ll}
I & 0
\end{array}\right] .
\end{gathered}
$$

The aim of this paper is to design a dynamic faulttolerant controller for the NCS (5), such that for all the possible data packet dropout and failures, the system (5) satisfies the following requirements.

(Q1) The closed-loop NCS (5) is asymptotically meansquare stable.

(Q2) Under the zero-initial condition, the output $z_{k}$ satisfies

$$
\sum_{k=0}^{\infty} \mathbb{E}\left\{\left\|z_{k}\right\|^{2}\right\}<\gamma^{2} \sum_{k=0}^{\infty} \mathbb{E}\left\{\left\|\omega_{k}\right\|^{2}\right\}+\delta^{2} \sum_{k=0}^{\infty} \mathbb{E}\left\{\left\|d_{k}\right\|^{2}\right\}
$$

for all nonzero $d_{k}, \omega_{k}$, where $\gamma, \delta>0$ are the scalars we will design.

\section{The Stability Analysis of NCS}

In this section, the stability analysis for the NCS (5) is discussed.

Lemma 4 (as in [28]). Let $W=W^{T}<0, H, N$ be matrices of appropriate dimensions, with $F$ satisfying $F^{T} F \leq I$, then $W+H F N+N^{T} F^{T} H^{T}<0$ holds, if and only if there exists a $\varepsilon>0$ such that $W+\varepsilon H H^{T}+\varepsilon^{-1} N^{T} N<0$.
Definition 5. The NCS with faults given by (5) with $\omega_{k}=$ $0, d_{k}=0$, is asymptotically mean-square stable, if for any initial state, $\lim _{k \rightarrow \infty} \mathbb{E}\left\{\left\|z_{k}\right\|^{2}\right\}=0$ holds.

Theorem 6. Given $\gamma>0, \delta>0$, the system (5) is asymptotically mean-square stable, and the output $z_{k}$ satisfies (7), if there exist matrices $P=P^{T}>0$, and $Q=Q^{T}>0$ satisfying

$$
\left[\begin{array}{ccccccccc}
-P & 0 & P \widetilde{A} & P \widetilde{A}_{d} & P \widetilde{D}_{1} & 0 & \sigma P \widetilde{B}_{1} & P \widetilde{B}_{2} & 0 \\
* & -I & \widetilde{C} & C_{d} & D_{1} & 0 & 0 & 0 & 0 \\
* & * & -P & 0 & 0 & \sigma \widetilde{A}_{2}^{T} P & 0 & 0 & Z^{T} \\
* & * & * & -Q & 0 & 0 & 0 & 0 & 0 \\
* & * & * & * & -\gamma^{2} I & 0 & 0 & 0 & 0 \\
* & * & * & * & * & -P & 0 & 0 & 0 \\
* & * & * & * & * & * & -\delta^{2} I & 0 & 0 \\
* & * & * & * & * & * & * & -\delta^{2} I & 0 \\
* & * & * & * & * & * & * & * & -Q^{-1}
\end{array}\right]<0 .
$$

Proof. Let $\Theta_{k}=\left[\eta_{k}^{T}, \eta_{k-1}^{T}, \ldots, \eta_{k-d}^{T}\right]^{T}$, for all nonzero $\eta_{k}$, consider the Lyapunov function $V_{k}\left(\Theta_{k}\right)=V_{1 k}+V_{2 k}$ with

$$
V_{1 k}=\eta_{k}^{T} P \eta_{k}, \quad V_{2 k}=\sum_{i=k-d}^{k-1} \eta_{i}^{T} Z^{T} Q Z \eta_{i} .
$$

The difference of $V_{k}$ is

$$
\begin{aligned}
\Delta V_{1 k}= & \mathbb{E}\left\{V_{1 k}\left(\Theta_{k+1}\right)\right\}-V_{1 k}\left(\Theta_{k}\right) \\
= & \eta_{k}^{T} \widetilde{A}^{T} P \widetilde{A}_{k}+\eta_{k}^{T} \widetilde{A}^{T} P \widetilde{A}_{d} Z \eta_{k-d}+\eta_{k}^{T} \widetilde{A}^{T} P \widetilde{B}_{2} d_{k} \\
& +\eta_{k}^{T} \widetilde{A}^{T} P \widetilde{D}_{1} \omega_{k}+\mathbb{E}\left\{\left(\theta_{k}-p\right)^{2}\right\} \eta_{k}^{T} \widetilde{A}_{2}^{T} P \widetilde{A}_{2} \eta_{k} \\
& +\mathbb{E}\left\{\left(\theta_{k}-p\right)^{2}\right\} \eta_{k}^{T} \widetilde{A}_{2}^{T} P \widetilde{B}_{1} d_{k}+\eta_{k-d}^{T} Z^{T} \widetilde{A}_{d}^{T} P \widetilde{A} \eta_{k} \\
& +\eta_{k-d}^{T} Z^{T} \widetilde{A}_{d}^{T} P \widetilde{A}_{d} Z \eta_{k-d}+\eta_{k-d}^{T} Z^{T} \widetilde{A}_{d}^{T} P \widetilde{B}_{2} d_{k} \\
& +\eta_{k-d}^{T} Z^{T} \widetilde{A}_{d}^{T} P \widetilde{D}_{1} \omega_{k}+\mathbb{E}\left\{\left(\theta_{k}-p\right)^{2}\right\} d_{k}^{T} \widetilde{B}_{1}^{T} P \widetilde{A}_{2} \eta_{k} \\
& +\mathbb{E}\left\{\left(\theta_{k}-p\right)^{2}\right\} d_{k}^{T} \widetilde{B}_{1}^{T} P \widetilde{B}_{1} d_{k}+d_{k}^{T} \widetilde{B}_{2}^{T} P \widetilde{A} \eta_{k} \\
& +d_{k}^{T} \widetilde{B}_{2}^{T} P \widetilde{A}_{d} Z \eta_{k-d}+d_{k}^{T} \widetilde{B}_{2}^{T} P \widetilde{B}_{2} d_{k} \\
& +d_{k}^{T} \widetilde{B}_{2}^{T} P \widetilde{D}_{1} \omega_{k}+\omega_{k}^{T} \widetilde{D}_{1}^{T} P \widetilde{A}_{k}+\omega_{k}^{T} \widetilde{D}_{1}^{T} P \widetilde{A}_{d} Z \eta_{k-d} \\
& +\omega_{k}^{T} \widetilde{D}_{1}^{T} P \widetilde{B}_{2} d_{k}+\omega_{k}^{T} \widetilde{D}_{1}^{T} P \widetilde{D}_{1} \omega_{k}-\eta_{k}^{T} P \eta_{k} \\
\Delta V_{2 k}= & \mathbb{E}\left\{V_{2 k}\left(\Theta_{k+1}\right)\right\}-V_{2 k}\left(\Theta_{k}\right) \\
= & \eta_{k}^{T}\left(Z^{T} Q Z\right) \eta_{k}-\eta_{k-d}^{T} Z^{T} Q Z \eta_{k-d} .
\end{aligned}
$$

For $\mathbb{E}\left\{\theta_{k}-p\right\}=0$, and denote $\mathbb{E}\left\{\left(\theta_{k}-p\right)^{2}\right\}=\sigma^{2}$, we have

$$
\begin{aligned}
\Delta V_{k} & =\left[\begin{array}{c}
\eta_{k} \\
Z \eta_{k-d} \\
d_{k} \\
\omega_{k}
\end{array}\right]^{T}\left[\begin{array}{cccc}
\Phi_{11} & \Phi_{12} & \Phi_{13} & \Phi_{14} \\
* & \Phi_{22} & \Phi_{23} & \Phi_{24} \\
* & * & \Phi_{33} & \Phi_{34} \\
* & * & * & \Phi_{44}
\end{array}\right]\left[\begin{array}{c}
\eta_{k} \\
Z \eta_{k-d} \\
d_{k} \\
\omega_{k}
\end{array}\right] \\
& <\zeta_{k}^{T} \Phi_{i j} \zeta_{k} \quad(i, j=1, \ldots, 4),
\end{aligned}
$$

where $\zeta_{k}=\left[\eta_{k}^{T}, \eta_{k-d}^{T} Z^{T}, d_{k}^{T}, \omega_{k}^{T}\right]^{T}, \Phi_{11}=\tilde{A}^{T} P \tilde{A}+$ $Z^{T} Q Z+\sigma^{2} \widetilde{A}_{2}^{T} P \widetilde{A}_{2}-P, \Phi_{12}=\widetilde{A}^{T} P \tilde{A}_{d}, \Phi_{13}=\tilde{A}^{T} P \widetilde{B}_{2}+$ $\sigma^{2} \widetilde{A}_{2}^{T} P \widetilde{B}_{1}, \Phi_{14}=\tilde{A}^{T} P \widetilde{D}_{1}, \Phi_{22}=\widetilde{A}_{d}^{T} P \widetilde{A}_{d}-Q, \Phi_{23}=$ 
$\widetilde{A}_{d}^{T} P \widetilde{B}_{2}, \Phi_{24}=\widetilde{A}_{d}^{T} P \widetilde{D}_{1}, \Phi_{33}=\widetilde{B}_{2}^{T} P \widetilde{B}_{2}+\sigma^{2} \widetilde{B}_{1}^{T} P \widetilde{B}_{1}, \Phi_{34}=$ $\widetilde{B}_{2}^{T} P \widetilde{D}_{1}, \Phi_{44}=\widetilde{D}_{1}^{T} P \widetilde{D}_{1}, *$ is implicitly defined by the fact that the matrix is symmetric.

When $\omega_{k}=0, d_{k}=0,(11)$ is rewritten as

$$
\begin{aligned}
& \Delta V_{k, \omega_{k}=d_{k}=0} \\
& =\left[\begin{array}{cc}
\tilde{A}^{T} P \tilde{A}+Z^{T} Q Z+\sigma^{2} \tilde{A}_{2}^{T} P \tilde{A}_{2}-P & \tilde{A}^{T} P \tilde{A}_{d} \\
\tilde{A}_{d}^{T} P \widetilde{A} & \tilde{A}_{d}^{T} P \widetilde{A}_{d}-Q
\end{array}\right] \\
& =\Pi \text {. }
\end{aligned}
$$

From (8) and the Schur complement theorem, $\Pi<0$ is arrived. Therefore, for all nonzero $\eta_{k}$, we have $\Delta V_{k}<0$, then the NCS (5) with sensor and actuator fault is asymptotically mean-square stable.

Next, For any nonzero $\omega_{k}, d_{k}$, it follows from (5), (8), and (11) that

$$
\begin{aligned}
& \mathbb{E}\left\{V\left(\Theta_{k+1}\right)\right\}-\mathbb{E}\left\{V\left(\Theta_{k}\right)\right\}+\mathbb{E}\left\{z_{k}^{T} z_{k}\right\} \\
&-\gamma^{2} \mathbb{E}\left\{\omega_{k}^{T} \omega_{k}\right\}-\delta^{2} \mathbb{E}\left\{d_{k}^{T} d_{k}\right\} \\
& \leq \mathbb{E}\{ \zeta_{k}^{T} \Phi_{i j} \zeta_{k}+\eta_{k}^{T} \widetilde{C}^{T} \widetilde{C} \eta_{k} \\
&+\eta_{k}^{T} \widetilde{C}^{T} C_{d} Z \eta_{k-d}+\eta_{k}^{T} \widetilde{C}^{T} D_{1} \omega_{k} \\
&+\eta_{k-d}^{T} Z^{T} C_{d}^{T} \widetilde{C} \eta_{k}+\eta_{k-d}^{T} Z^{T} C_{d}^{T} C_{d} Z \eta_{k-d} \\
&+\eta_{k-d}^{T} Z^{T} C_{d}^{T} D_{1} \omega_{k}+\omega_{k}^{T} D_{1}^{T} \widetilde{C} \eta_{k} \\
&+\omega_{k}^{T} D_{1}^{T} C_{d} Z \eta_{k-d}+\omega_{k}^{T} D_{1}^{T} D_{1} \omega_{k} \\
&\left.-\gamma^{2} \omega_{k}^{T} \omega_{k}-\delta^{2} d_{k}^{T} d_{k}\right\} .
\end{aligned}
$$

Then, we have

$$
\begin{gathered}
\mathbb{E}\left\{V\left(\Theta_{k+1}\right)\right\}-\mathbb{E}\left\{V\left(\Theta_{k}\right)\right\}+\mathbb{E}\left\{z_{k}^{T} z_{k}\right\} \\
-\gamma^{2} \mathbb{E}\left\{\omega_{k}^{T} \omega_{k}\right\}-\delta^{2} \mathbb{E}\left\{d_{k}^{T} d_{k}\right\}<0 .
\end{gathered}
$$

Now, summing (14) from 0 to $\infty$ with respect to $k$ yields

$$
\begin{aligned}
\sum_{k=0}^{\infty} \mathbb{E}\left\{z_{k}^{T} z_{k}\right\}< & \gamma^{2} \sum_{k=0}^{\infty} \mathbb{E}\left\{\omega_{k}^{T} \omega_{k}\right\}+\delta^{2} \sum_{k=0}^{\infty} \mathbb{E}\left\{d_{k}^{T} d_{k}\right\} \\
& +\mathbb{E}\left\{V_{0}\right\}-\mathbb{E}\left\{V_{\infty}\right\} .
\end{aligned}
$$

Since the system (5) is asymptotically mean-square stable, we can get that the following inequality:

$$
\sum_{k=0}^{\infty} \mathbb{E}\left\{z_{k}^{T} z_{k}\right\}<\gamma^{2} \sum_{k=0}^{\infty} \mathbb{E}\left\{\omega_{k}^{T} \omega_{k}\right\}+\delta^{2} \sum_{k=0}^{\infty} \mathbb{E}\left\{d_{k}^{T} d_{k}\right\}
$$

holds under the zero initial condition. The proof is thus, complete.

\section{Robust $H_{\infty}$ Controller Design}

In this section, a theorem will be proposed to solve the controller design problem for stochastic state-delay NCS (5).

Theorem 7. Given a scalar $\gamma>0, \delta>0$, the system (5) is asymptotically mean-square stable, and the controlled output $z_{k}$ satisfies the $H_{\infty}$ constraints (7), if there exist real scalars $\varepsilon>$ 0 , and matrices $S=S^{T}>0, Q=Q^{T}>0$, and $R=R^{T}>$ 0 , and real matrices $W_{1}, W_{2}$, and $W_{3}$, such that the following inequality holds:

$$
\left[\Omega_{i j}\right]<0, \quad i, j=1, \ldots, 16,
$$

where $\Omega_{11}=-S^{-1}, \Omega_{12}=-I, \Omega_{14}=A+p B W_{3}, \Omega_{15}=$ $A, \Omega_{16}=A_{d}, \Omega_{17}=D_{0}, \Omega_{1,10}=-\sigma B, \Omega_{1,12}=(1-$ p) $B, \Omega_{1,15}=H, \Omega_{21}=-I, \Omega_{22}=-R, \Omega_{24}=R A+p W_{2} C_{0}+$ $W_{1}, \Omega_{25}=R A+p W_{2} C_{0}, \Omega_{26}=R A_{d}, \Omega_{27}=R D_{0}, \Omega_{2,10}=$ $-\sigma R B, \Omega_{2,11}=-\sigma W_{2}, \Omega_{2,12}=(1-p) R B, \Omega_{2,13}=$ $(1-p) W_{2}, \Omega_{2,15}=R H, \Omega_{33}=-I, \Omega_{34}=C, \Omega_{35}=$ $C, \Omega_{36}=C_{d}, \Omega_{37}=D_{1}, \Omega_{41}=\Omega_{14}^{T}, \Omega_{42}=\Omega_{24}^{T}, \Omega_{43}=$ $C^{T}, \Omega_{44}=-S, \Omega_{45}=-S, \Omega_{48}=\sigma W_{3}^{T} B^{T}, \Omega_{49}=$ $\sigma W_{3}^{T} B^{T} R+\sigma C_{0}^{T} W_{2}^{T}, \Omega_{4,14}=I, \Omega_{4,16}=\epsilon N^{T}, \Omega_{51}=$ $A^{T}, \Omega_{52}=\Omega_{25}^{T}, \Omega_{53}=C^{T}, \Omega_{54}=-S, \Omega_{55}=-R, \Omega_{59}=$ $\sigma C_{0}^{T} W_{2}^{T}, \Omega_{5,14}=Q, \Omega_{5,16}=\epsilon N^{T}, \Omega_{61}=A_{d}^{T}, \Omega_{61}=$ $A_{d}^{T} R, \Omega_{63}=C_{d}^{T}, \Omega_{66}=-Q, \Omega_{71}=D_{0}^{T}, \Omega_{72}=$ $D_{0}^{T} R, \Omega_{73}=D_{1}^{T}, \Omega_{77}=-\gamma^{2} I, \Omega_{84}=\Omega_{48}^{T}, \Omega_{88}=$ $-S, \Omega_{89}=-S, \Omega_{94}=\Omega_{49}^{T}, \Omega_{95}=\Omega_{59}^{T}, \Omega_{99}=-R, \Omega_{10,1}=$ $-\sigma B^{T}, \Omega_{10,2}=-\sigma B^{T} R, \Omega_{10,10}=\Omega_{11,11}=\Omega_{12,12}=\Omega_{13,13}=$ $-\delta^{2} I, \Omega_{11,2}=\Omega_{2,11}^{T}, \Omega_{12,1}=\Omega_{12,1}^{T}, \Omega_{12,2}=\Omega_{2,12}^{T}, \Omega_{13,2}=$ $\Omega_{2,13}^{T}, \Omega_{14,4}=I, \Omega_{14,5}=Q^{T}, \Omega_{14,14}=-I, \Omega_{15,1}=$ $H^{T}, \Omega_{15,2}=H^{T} R, \Omega_{15,15}=\Omega_{16,16}=-\varepsilon I, \Omega_{16,4}=\Omega_{16,5}=$ $\epsilon N$, the rest of matrix entries are zero.

The fault-tolerant controller parameters are

$$
\begin{aligned}
& A_{f}=X_{12}^{-1}\left[\left(W_{1}-p R B W_{3}\right) S^{-1}\left(Y_{12}^{T}\right)^{-1}\right], \\
& B_{f}=X_{12}^{-1} W_{2}, \quad C_{f}=W_{3} S^{-1}\left(Y_{12}^{T}\right)^{-1} .
\end{aligned}
$$

Proof. The system (5) is a parameter-dependent system. By Lemma $4,(8)$ is rewritten as

$$
\left[\begin{array}{ccccccccccc}
-P & 0 & P \tilde{A}_{1} & P \widetilde{A}_{d} & P \widetilde{D}_{1} & 0 & \sigma P \widetilde{B}_{1} & P \widetilde{B}_{2} & 0 & P \tilde{H} & 0 \\
* & -I & \widetilde{C} & C_{d} & D_{1} & 0 & 0 & 0 & 0 & 0 & 0 \\
* & * & -P & 0 & 0 & \sigma \widetilde{A}_{2}^{T} P & 0 & 0 & Z^{T} & 0 & \epsilon \tilde{N}^{T} \\
* & * & * & -Q & 0 & 0 & 0 & 0 & 0 & 0 & 0 \\
* & * & * & * & -\gamma^{2} I & 0 & 0 & 0 & 0 & 0 & 0 \\
* & * & * & * & * & -P & 0 & 0 & 0 & 0 & 0 \\
* & * & * & * & * & * & -\delta^{2} I & 0 & 0 & 0 & 0 \\
* & * & * & * & * & * & * & -\delta^{2} I & 0 & 0 & 0 \\
* & * & * & * & * & * & * & * & -Q^{-1} & 0 & 0 \\
* & * & * & * & * & * & * & * & * & -\epsilon I & 0 \\
* & * & * & * & * & * & * & * & * & * & -\epsilon I
\end{array}\right]<0 .
$$


Next, partition $P$ and $P^{-1}$ as

$$
P=\left[\begin{array}{cc}
R & X_{12} \\
X_{12}^{T} & X_{22}
\end{array}\right], \quad P^{-1}=\left[\begin{array}{cc}
S^{-1} & Y_{12} \\
Y_{12}^{T} & Y_{22}
\end{array}\right]
$$

Define

$$
T_{1}=\left[\begin{array}{cc}
S^{-1} & I \\
Y_{12}^{T} & 0
\end{array}\right], \quad T_{2}=\left[\begin{array}{cc}
I & R \\
0 & X_{12}^{T}
\end{array}\right] .
$$

Obviously, we have $P T_{1}=T_{2}, T_{1}^{T} P T_{1}=T_{1}^{T} T_{2}$. Performing the congruence transformation $\operatorname{diag}\left\{T_{1}^{T}, \mathrm{I}, T_{1}^{T}, \mathrm{I}, \mathrm{I}, T_{1}^{T} I, \mathrm{I}\right.$, $\left.T_{1}^{T}, \mathrm{I}, \mathrm{I}\right\}$ to (19), we obtain the following:

$$
\left[\Lambda_{i, j}\right]<0, \quad i, j=1, \ldots, 16
$$

where $\Lambda_{11}=-S^{-1}, \Lambda_{12}=-I, \Lambda_{14}=A S^{-1}+p B C_{f} Y_{12}^{T}$, $\Lambda_{15}=A, \Lambda_{16}=A_{d}, \Lambda_{17}=D_{0}, \Lambda_{1,10}=-\sigma B, \Lambda_{1,12}=$ $(1-p) B, \Lambda_{1,15}=H, \Lambda_{21}=-I, \Lambda_{22}=-R, \Lambda_{24}=(R A+$ $\left.p X_{12} B_{f} C_{0}\right) S^{-1}+\left(p R B C_{f}+X_{12} A_{f}\right) Y_{12}^{T}, \Lambda_{25}=R A+p X_{12} B_{f} C_{0}$, $\Lambda_{26}=R A_{d}, \Lambda_{27}=R D_{0}, \Lambda_{2,10}=-\sigma R B, \Lambda_{2,11}=-\sigma X_{12} B_{f}$, $\Lambda_{2,12}=(1-p) R B, \Lambda_{2,13}=(1-p) X_{12} B_{f}, \Lambda_{2,15}=R H$, $\Lambda_{33}=-I, \Lambda_{34}=C S^{-1}, \Lambda_{35}=C, \Lambda_{36}=C_{d}, \Lambda_{37}=D_{1}$, $\Lambda_{41}=\Lambda_{41}^{T}, \Lambda_{42}=\Lambda_{42}^{T}, \Lambda_{43}=S^{-1} C^{T}, \Lambda_{44}=-S^{-1}, \Lambda_{45}=$ $-I, \Lambda_{48}=\sigma Y_{12} C_{f}^{T} B^{T}, \Lambda_{49}=\sigma Y_{12} C_{f}^{T} B^{T} R+\sigma S^{-1} C_{0}^{T} B_{f}^{T} X_{12}^{T}$, $\Lambda_{4,14}=S^{-1}, \Lambda_{4,16}=\epsilon S^{-1} N^{T}, \Lambda_{51}=A^{T}, \Lambda_{52}=\Lambda_{25}^{T}$, $\Lambda_{53}=C^{T}, \Lambda_{54}=-S, \Lambda_{55}=-R, \Lambda_{59}=\sigma C_{0}^{T} B_{f}^{T} X_{12}^{T}$, $\Lambda_{5,12}=I, \Lambda_{5,16}=\epsilon N^{T}, \Lambda_{61}=A_{d}^{T}, \Lambda_{62}=A_{d}^{T} R, \Lambda_{63}=C_{d}^{T}$, $\Lambda_{66}=-Q, \Lambda_{71}=D_{0}^{T}, \Lambda_{72}=D_{0}^{T} R, \Lambda_{73}=D_{1}^{T}, \Lambda_{77}=-\gamma^{2} I$, $\Lambda_{84}=\Lambda_{48}^{T}, \Lambda_{88}=-S^{-1}, \Lambda_{89}=-I, \Lambda_{94}=\Lambda_{49}^{T}, \Lambda_{95}=\Lambda_{59}^{T}$, $\Lambda_{99}=-R, \Lambda_{10,1}=-\sigma B^{T}, \Lambda_{10,2}=-\sigma B^{T} R, \Lambda_{11,2}=-\sigma X_{12} B_{f}$, $\Lambda_{12,1}=(1-p) B^{T}, \Lambda_{12,2}=(1-p) B^{T} R, \Lambda_{13,2}=(1-p) X_{12} B_{f}$, $\Lambda_{14,4}=S^{-1}, \Lambda_{14,5}=I, \Lambda_{10,10}=\Lambda_{11,11}=\Lambda_{12,12}=\Lambda_{13,13}=$ $-\delta^{2} I, \Lambda_{14,14}=-Q^{-1}, \Lambda_{15,15}=\Lambda_{16,16}=-\epsilon I, \Lambda_{15,1}=H^{T}$, $\Lambda_{15,2}=H^{T} R, \Lambda_{16,4}=\epsilon N S^{-1}, \Lambda_{16,5}=\epsilon N$, the rest of matrix entries are zero.

Applying the congruence transformation $\operatorname{diag}\{\mathrm{I}, \mathrm{I}, \mathrm{I}, \mathrm{S}$, I, I, I, S, I, I, I, I, I, Q, I, I\} to (22) again, then (17) is achieved. Therefore, by Theorem 6 , the desired result follows immediately.

Next, we will design the scalars $\gamma$ and $\delta$ by solving the optimization problem,

$$
\min _{\epsilon, S, R, Q>0, W_{1}, W_{2}, W_{3}} \gamma, \delta, \quad \text { subject to }(17)
$$

Using the robust control toolbox, we will get the optimal value of $\gamma$ and $\delta$, and the proof is thus, complete.

Remark 8. From Theorems 6 and 7, we know if (17) is feasible, $I-R S^{-1}=X_{12} Y_{12}^{T}<0$, hence, the square and nonsingular matrices $X_{12}$ and $Y_{12}$ can be always found (as in [29]). Then, the fault-tolerant controller parameters (18) are obtained.

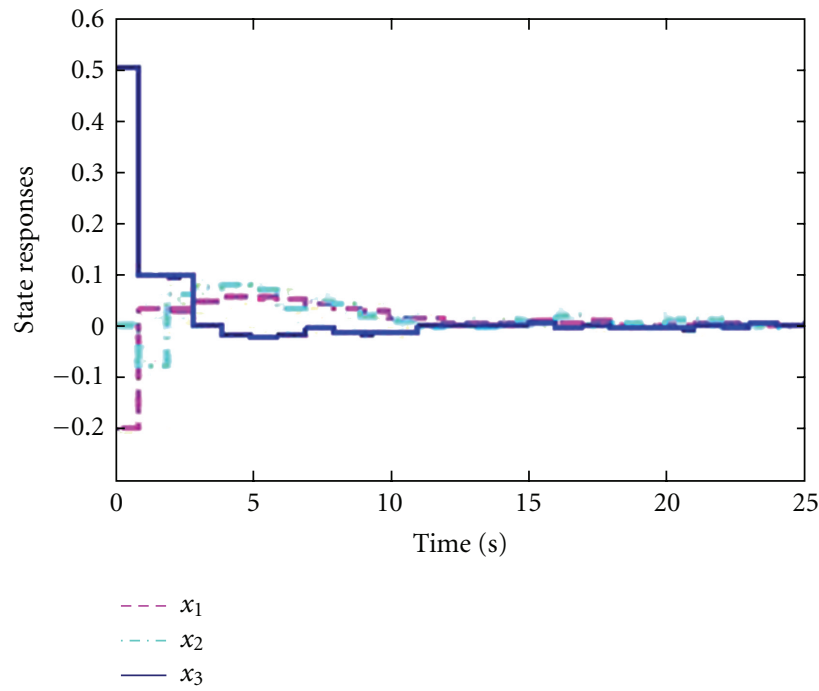

Figure 2: State responses under the fault-tolerant controller.

\section{Simulation Example}

Consider the system (1) with parameters (as in [22]) as follows:

$$
\begin{gathered}
A=\left[\begin{array}{ccc}
-0.3 & 0 & -0.3 \\
0 & 0.6 & 0.2 \\
0.5 & 0 & 0.7
\end{array}\right], \quad A_{d}=\left[\begin{array}{ccc}
-0.1 & 0 & 0 \\
0 & 0.1 & 0 \\
0 & 0 & 0.2
\end{array}\right], \\
B=\left[\begin{array}{l}
0 \\
1 \\
2
\end{array}\right], \quad D_{0}=\left[\begin{array}{c}
0.5 \\
0 \\
0.2
\end{array}\right], \quad H=\left[\begin{array}{c}
0.5 \\
0.5 \\
1
\end{array}\right], \\
C_{0}=\left[\begin{array}{lll}
1 & 1 & 2
\end{array}\right], \quad C=\left[\begin{array}{lll}
0.1 & 0 & 0
\end{array}\right], \\
C_{d}=\left[\begin{array}{lll}
0.1 & 0 & 0
\end{array}\right], \quad N=\left[\begin{array}{lll}
0.1 & 0 & 0
\end{array}\right], \quad D_{1}=0.1 .
\end{gathered}
$$

Choose the same parameters as [22], packet loss rate is $p=$ 0.1 , delay constant is $d=3$. With the method in [22], the optimal performance $\gamma$ is 3.3339. Using the proposed method in this paper, the optimal performance $\gamma$ is 1.3614 , which means the smaller performance has been obtained.

Next, let the packet loss rate and fault probability $p=0.3$, delay constant is $d=2$, under the initial condition $x(0)=$ $\left[\begin{array}{lll}-0.2 & 0 & 0.5\end{array}\right]^{T}, \gamma=1.6845$, and $\delta=2.0753$, the faulttolerant controller parameters are designed by Theorem 7 as follows.

$$
\begin{gathered}
A_{f}=\left[\begin{array}{ccc}
0.1357 & 2.4132 & -0.9861 \\
-2.1036 & -1.0003 & -0.0584 \\
-1.5372 & -0.4739 & -2.9271
\end{array}\right], \\
B_{f}=\left[\begin{array}{c}
-0.0697 \\
0.7264 \\
-0.0983
\end{array}\right], \quad C_{f}=\left[\begin{array}{lll}
-0.1016 & -0.0712 & 0.2598
\end{array}\right] .
\end{gathered}
$$

From Figure 2, it can be seen that state responses under the designed controller can stabilize the NCS with data packet loss, probabilistic actuator failures, and/or sensor 
failures, which can illustrate the effectiveness of the proposed method.

\section{Conclusion}

Motivated by robust FTC problem over networks, a new stochastic NCS model with fault is addressed, which includes the state-delay, model uncertainty, disturbance, random packet dropout, probabilistic sensors failures, and actuators failures. The random packet dropout in both S-C link and C-A link, the sensor failure and the actuator failure are described as a binary random variable. The sufficient condition for asymptotical mean-square stability of the NCS has been derived and the closed-loop NCS satisfies $H_{\infty}$ performance constraints. Finally, by solving a linear matrix inequality, the fault tolerant controller is designed.

\section{Acknowledgments}

The authors would like to acknowledge the National Natural Science Foundation of China under Grant (61174044), and the Shandong Province Natural Science Foundation under Grant (ZR2010FM016). The authors also wish to thank the reviewers for their valuable suggestions.

\section{References}

[1] W. Zhang, M. Branicky, and S. Phillips, "Stability of networked control systems," IEEE Control Systems Magazine, vol. 21, no. 1, pp. 84-97, 2001.

[2] G. Walsh, H. Ye, and L. Bushnell, "Stability analysis of networked control systems," IEEE Transactions on Control Systems Technology, vol. 10, no. 3, pp. 438-446, 2002.

[3] J. P. Hespanha, P. Naghshtabrizi, and Y. Xu, "A survey of recent results in networked control systems," Proceedings of the IEEE, vol. 95, no. 1, Article ID 4118453, pp. 138-162, 2007.

[4] L. Zhang, Y. Shi, T. Chen, and B. Huang, "A new method for stabilization of networked control systems with random delays," IEEE Transactions on Automatic Control, vol. 50, no. 8, pp. 1177-1181, 2005.

[5] Y. Shi and B. Yu, "Output feedback stabilization of networked control systems with random delays modeled by Markov chains," IEEE Transactions on Automatic Control, vol. 54, no. 7, pp. 1668-1674, 2009.

[6] H. Zhang, Y. Shi, and A. S. Mehr, "Robust energy-to-peak filtering for networked systems with time-varying delays and randomly missing data," IET Control Theory and Applications, vol. 4, no. 12, pp. 2921-2936, 2010.

[7] K. C. Walker, Y. J. Pan, and J. Gu, "Bilateral teleoperation over networks based on stochastic switching approach," IEEE/ASME Transactions on Mechatronics, vol. 14, no. 5, pp. 539-554, 2009.

[8] H. Zhang, Y. Shi, and S. A. Mehr, "Robust static output feedback control and remote PID design for networked motor systems," IEEE Transactions on Industrial Electronics, vol. 58, no. 12, pp. 5396-5405, 2011.

[9] H. Zhang, Y. Shi, S. A. Mehr, and H. Huang, "Robust FIR equalization for time-varying communication channels with intermittent observations via an LMI approach," Signal Processing, vol. 91, no. 7, pp. 1651-1658, 2011.
[10] H. Zhang, Y. Shi, and A. S. Mehr, "Robust weighted $\mathrm{H}_{\infty}$ filtering for networked systems with intermitted measurements of multiple sensors," International Journal of Adaptive Control and Signal Processing, vol. 25, pp. 313-330, 2011.

[11] M. Blanke, M. Kinnaert, J. Lunze, and M. Staroswiecki, Diagnosis and Fault-Tolerant Control, Springer, Berlin, Germany, 2003.

[12] J. Chen and R. J. Patton, Robust Model-Based Fault Diagnosis for Dynamic Systems, Kluwer Academic, Boston, Mass, USA, 1999.

[13] H. Noura, D. Theilliol, J.-C. Ponsart, and A. Chamseddine, Fault-Tolerant Control Systems, Springer, London, UK, 2009.

[14] Y. M. Zhang and J. Jiang, "Bibliographical review on reconfigurable fault-tolerant control systems," Annual Reviews in Control, vol. 32, no. 2, pp. 229-252, 2008.

[15] S. X. Ding, P. Zhang, C. I. Chihaia et al., "Advanced design scheme for fault tolerant distributed networked control systems," in Proceedings of the 17th IFAC World Congress, pp. 13569-13574, Seoul City, Korea, 2008.

[16] C. X. Yang, Z. H. Guan, and J. Huang, "Stochastic fault tolerant control of networked control systems," Journal of the Franklin Institute, vol. 346, no. 10, pp. 1006-1020, 2009.

[17] Z. Huo and H. Fang, "Research on robust fault-tolerant control for networked control system with packet dropout," Journal of Systems Engineering and Electronics, vol. 18, no. 1, pp. 76-82, 2007.

[18] S. Li, D. Sauter, C. Aubrun, and J. Yamé, "Stability guaranteed active fault-tolerant control of networked control systems," Journal of Control Science and Engineering, vol. 2008, Article ID 189064, 2008.

[19] D. Xie and Y. Wu, "Fault-tolerant control of networked control systems with packet dropouts: switched system approach," in Proceedings of the Chinese Control and Decision Conference (CCDC '09), pp. 5577-5581, June 2009.

[20] Z. H. Huo, Y. Zheng, and C. Xu, "A robust fault-tolerant control strategy for networked control systems," Journal of Network and Computer Applications, vol. 34, pp. 708-714, 2011.

[21] X. He and D. H. Zhou, "Robust $H_{\infty}$ filtering for timedelay systems with probabilistic sensor faults," IEEE Signal Processing Letters, vol. 16, no. 5, pp. 442-445, 2009.

[22] F. Yang, Z. Wang, D. W. C. Ho, and M. Gani, "Robust $H_{\infty}$ control with missing measurements and time delays," IEEE Transactions on Automatic Control, vol. 52, no. 9, pp. 16661672, 2007.

[23] E. Tian, D. Yue, and C. Peng, "Reliable control for networked control systems with probabilistic actuator fault and random delays," Journal of the Franklin Institute, vol. 347, no. 10, pp. 1907-1926, 2010.

[24] E. Tian, D. Yue, and C. Peng, "Brief Paper: reliable control for networked control systems with probabilistic sensors and actuators faults," IET Control Theory and Applications, vol. 4, no. 8, pp. 1478-1488, 2010.

[25] C. Peng, T. C. Yang, and E. Tian, "Robust fault-tolerant control of networked control systems with stochastic actuator failure," IET Control Theory and Applications, vol. 4, no. 12, pp. 30033011, 2010.

[26] H. Zhang, A. S. Mehr, and Y. Shi, "Improved robust energy-topeak filtering for uncertain linear systems," Signal Processing, vol. 90, no. 9, pp. 2667-2675, 2010. 
[27] H. Zhang, Y. Shi, and A. S. Mehr, "Robust non-fragile dynamic vibration absorbers with uncertain factors," Journal of Sound and Vibration, vol. 330, no. 4, pp. 559-566, 2011.

[28] Y. Ji, H. J. Chizeck, X. Feng, and K. A. Loparo, "Stability and control of discrete-time jump linear systems," Control, Theory and Advanced Technology, vol. 7, no. 2, pp. 247-270, 1991.

[29] C. Scherer, P. Gahinet, and M. Chilali, "Multiobjective outputfeedback control via LMI optimization," IEEE Transactions on Automatic Control, vol. 42, no. 7, pp. 896-911, 1997. 

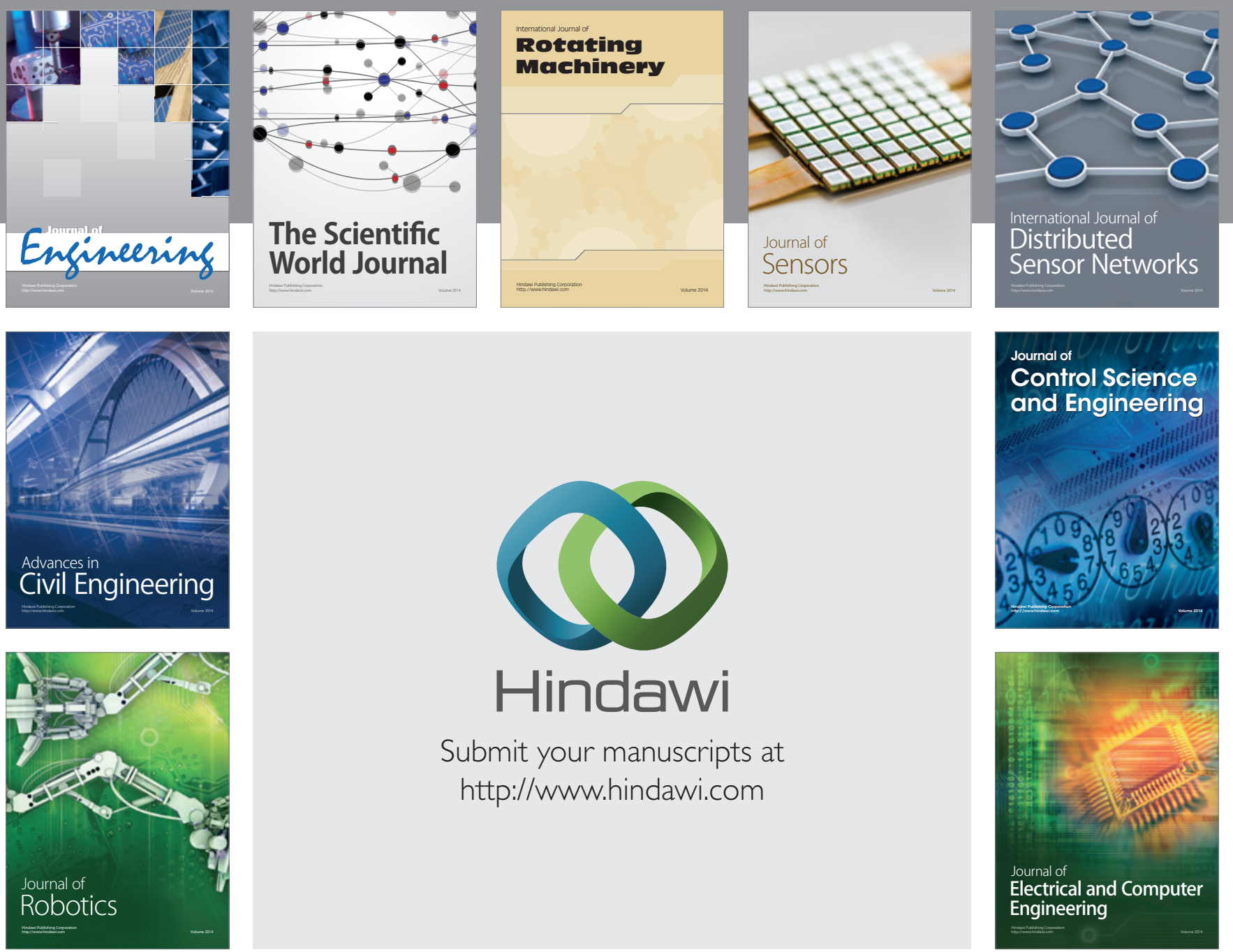

Submit your manuscripts at

http://www.hindawi.com
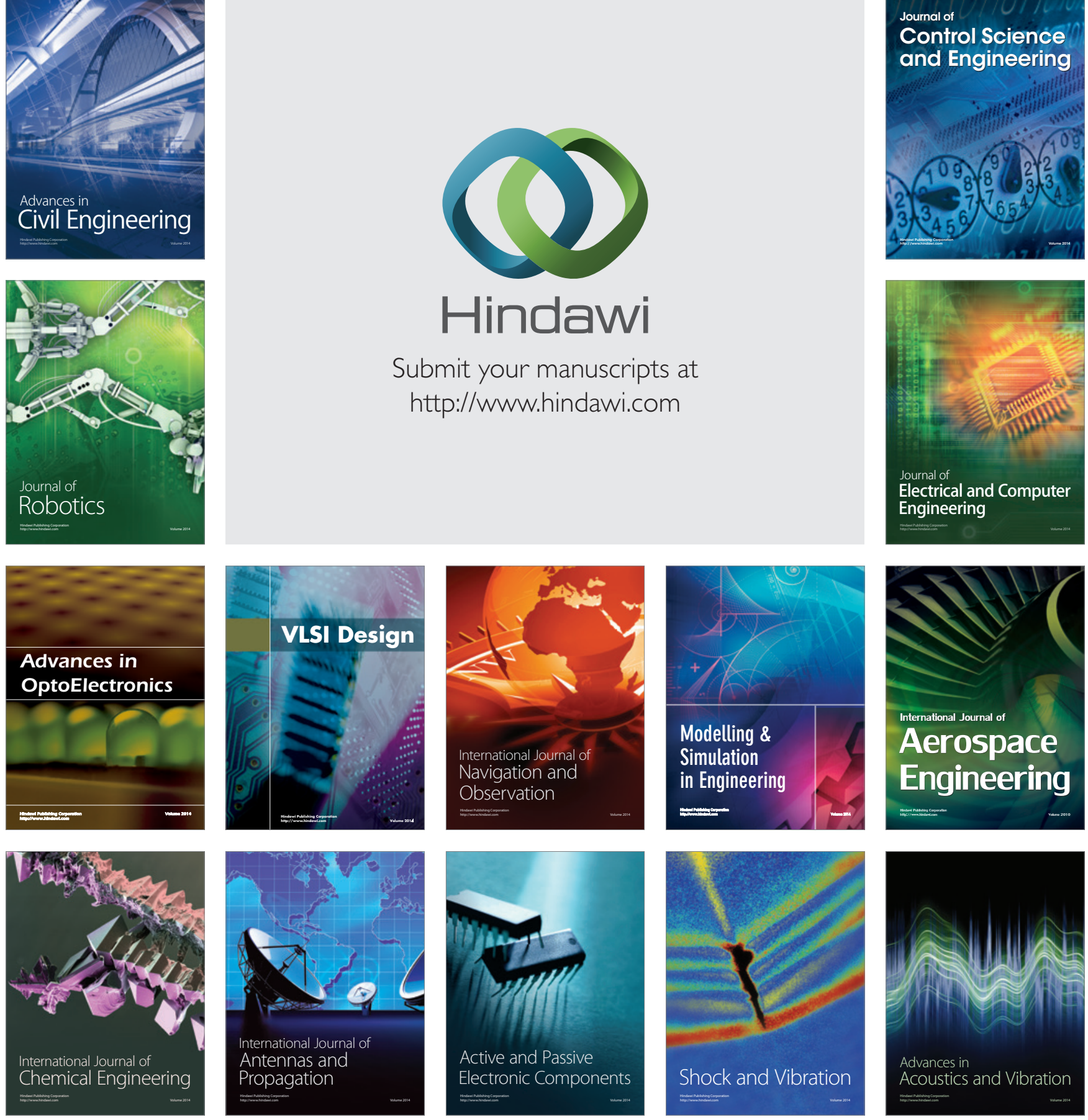